\title{
Nucleation of organic semiconductors on inert substrates
}

\author{
Stijn Verlaak, Soeren Steudel, and Paul Heremans \\ IMEC, Kapeldreef 75, B-3001 Leuven, Belgium \\ and Department of Electrical Engineering, Katholieke Universiteit Leuven, Belgium \\ Dimitri Janssen \\ IMEC, Kapeldreef 75, B-3001 Leuven, Belgium \\ and Department of Chemistry, Katholieke Universiteit Leuven, Belgium
}

Michael S. Deleuze

Limburgs Universitair Centrum, Department SBG, Universitaire Campus Building D, B-3590 Diepenbeek, Belgium

(Received 4 July 2003; published 18 November 2003)

\begin{abstract}
We have adapted the microscopic theory of nucleation for the epitaxial growth of inorganic materials to the nucleation of organic small molecules on an inert substrate like the gate dielectric of an organic thin-film transistor. The parameters required to explore the model were calculated with the standard MM3 force field and also include experimentally determined vapor pressure data, as well as film growth data. Sufficient agreement is found between the experimentally determined equilibrium crystal shape and heats of sublimation on the one hand and the calculated parameters on the other hand. The growth of pentacene, tetracene, and perylene on inert substrates has been studied in terms of this theory, especially focusing on the two-dimensional (2D) to 3D nucleation transition. It is demonstrated that 3D nucleation leads to ill-connected grains, while 2D nucleated grains form continuous films suitable for charge transport. The analysis of this transition allows for the experimental determination of the molecule-substrate interactions for a given molecule on a given surface. It was found that the deposition conditions for 2D growth shift to less favorable substrate temperatures and deposition rates as the difference between interlayer interactions and molecule-substrate interactions increase and the intralayer interactions decrease. Moreover, those interactions affect the nucleation rate and therefore the ultimate 2D grain size that can be obtained.
\end{abstract}

DOI: 10.1103/PhysRevB.68.195409

PACS number(s): 68.55.- a, 64.60.Qb, 31.70.Ks

\section{INTRODUCTION}

Organic thin-film transistors (OTFT's) offer a promising technology for low-cost large-area electronics on flexible substrates. Potential applications are in addressing circuits of liquid crystal or organic light-emitting diode displays and radio-frequency identification tags, among others. ${ }^{1}$ For applications where current driving capacity and switching speed are important, (poly)crystalline sublimed organic smallmolecule thin films have advantages over solution-processed films because of their higher charge carrier mobility. The mobility of polycrystalline molecular thin films is intrinsically limited by the charge carrier mobility of the molecular bulk crystal. Despite the fact that many organic bulk crystals display charge carrier drift mobilities larger than $1 \mathrm{~cm}^{2} / \mathrm{V} \mathrm{s}$ at room temperature, ${ }^{2,3}$ so far only a few materials, like pentacene, have demonstrated thin-film charge carrier mobilities approaching their bulk crystal values. ${ }^{4}$ Moreover, crystallinity and morphology of the thin film have proven to be crucial in approaching maximum mobilities. ${ }^{5}$ Apparently, exploitations of a molecular organic semiconductor in active layers will not so much be limited by the intrinsic charge carrier mobility in the material, but rather by its film growth properties.

Here, we study the nucleation of pentacene, tetracene, and perylene on inert substrates-i.e., a substrate developing only weak interactions with the physisorbed molecules. Typical examples of such substrates are gate dielectrics of OT-
FT's. The forementioned molecules crystallize in a layered structure with the layers parallel to the inert substrate and with a herringbone or sandwich herringbone arrangement within the layers and are ideal candidates for charge transport layers in OTFT's.

It has been found that organic thin-film growth closely mimics the epitaxial growth of inorganic materials. ${ }^{6}$ Concepts from film growth of inorganic materials have been used to interpret the growth of organic thin films on inert substrates. ${ }^{7}$ We adapt the atomistic theory of nucleation as a formal framework to interpret our results. Our theory predicts that the transition from two-dimensional to threedimensional nucleation crosses the range of growth conditions that is usually applied to prepare polycrystalline organic thin films. Three-dimensional nuclei form illconnected grains and lead to discontinuous films. These films, obviously, are less suited for applications in OTFT's, since film continuity is a basic requirement for charge transport layers.

\section{MODEL}

In an organic molecular beam deposition (OMBD) tool, film growth requires that the vapor phase and crystalline phase deposited on the substrate not be in thermodynamic equilibrium. Equilibrium in systems with constant pressure and constant temperature is thermodynamically required to be at the minimum of the Gibbs free energy $G$ : i.e., $d G$ 

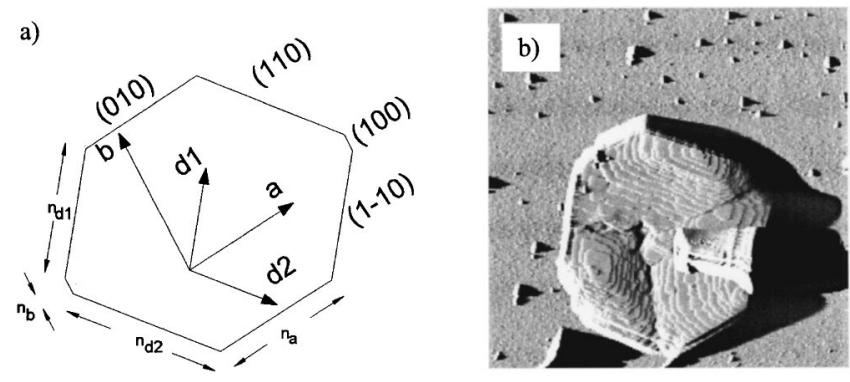

FIG. 1. (a) shows the equilibrium shape in the $a b$ plane of a pentacene nucleus bounded by (010), (110), (100), (1-10), and (001) planes, using the parameters for pentacene (2) in Tables I and II. Dimensions are given in number of unit cells. The nucleus is $n_{c}$ unit cells high. The absolute dimensions of the nucleus for a certain supersaturation follow from optimization of Eq. (1) or from the Wulff rule. (b) shows an atomic force microscopy picture (5 $\times 5 \mu \mathrm{m}^{2}$ ) of an isolated pentacene grain grown at very low supersaturations.

$=0$. The equilibrium between a vapor $(v)$ and an infinitely large crystal $(c)$, both at the same temperature and pressure, follows from the condition that the minimal Gibbs free energy not change when transferring a molecule from one phase to the other: i.e., $(\delta G / \delta n)_{v}=(\delta G / \delta n)_{c}$ or $\mu_{v}$ $=\mu_{c}$. Here, the chemical potential $\mu=\delta G / \delta n$ expresses the work which has to be done in order to change the number of particles in the phase by 1 . If the ambient vapor phase is supersaturated, the (positive) difference $\Delta \mu$ between the chemical potential of the vapor phase and the infinitely large crystal (called "supersaturation") appears as the thermodynamic driving force of the crystallization process.

When at supersaturation a molecule is transferred from the vapor to a solid nucleus with finite size rather than to an infinitely large crystal, the energy which is released to the surrounding does not equal $\Delta \mu$ but is lowered due to finite size effects. ${ }^{8}$ The energy required for the formation of a finite nucleus (shortly, the energy of formation) can then be expressed as

$$
\begin{aligned}
\Delta G= & -n_{a b} n_{c} m_{u} \Delta \mu+\left[2 n_{b} n_{c} \psi_{100}+2 n_{a} n_{c} \psi_{010}\right. \\
& \left.+2 n_{d 1} n_{c} \psi_{1-10}+2 n_{d 2} n_{c} \psi_{110}+n_{a b} \psi_{001}+n_{a b} \psi_{s}\right]
\end{aligned}
$$

where $n_{a}, n_{b}, n_{c}, n_{d 1}$, and $n_{d 2}$ are the number of unit cells along the edges of the nucleus parallel to the directions indicated by the subscript, as clarified in Fig. 1(a), $m_{u}$ is the number of molecules per unit cell (i.e., $m_{u}=2$ for pentacene and tetracene and $m_{u}=4$ for the perylene polymorph under consideration), and $n_{a b}$ is the total number of unit cells within one monolayer in the $a b$ plane of the nucleus in Fig. 1 (a) and is fully determined by $n_{a}, n_{b}, n_{d 1}$ and $n_{d 2}$ :

$$
\begin{aligned}
n_{a b} \approx & n_{a}\left(\frac{n_{b}+n_{d 1}+n_{d 2}}{2}\right)+n_{b}\left(\frac{n_{a}+n_{d 1}+n_{d 2}}{2}\right) \\
& +n_{d 1}\left(\frac{n_{a}+n_{b}+2 n_{d 2}}{2}\right)+n_{d 2}\left(\frac{n_{a}+n_{b}+2 n_{d 1}}{2}\right) .
\end{aligned}
$$

Equation (2) neglects the linear terms $+n_{d 1}+n_{d 2}$ to ease calculation. It is assumed and validated later that the nucleus is bounded by ten surfaces, including the top surface and the substrate interface. The first term of Eq. (1) then expresses the energy released upon transferring $m_{u} n_{a b} n_{c}$ molecules from the vapor to an infinitely large crystal. The second term corrects for the finite size of a nucleus containing $m_{u} n_{a b} n_{c}$ molecules in terms of the specific free surface energies per unit cell, which are defined as half the work spent per unit cell to create two new surfaces (i.e., the work to create one surface) by splitting an infinitely large crystal, ${ }^{8}$

$$
\begin{aligned}
& \psi_{100}=\frac{2 \psi_{a}+\psi_{d 1}+\psi_{d 2}}{2}, \\
& \psi_{010}= \frac{2 \psi_{b}+\psi_{d 1}+\psi_{d 2}}{2}, \\
& \psi_{110}= \frac{2 \psi_{a}+2 \psi_{b}+2 \psi_{d 1}}{2}, \\
& \psi_{1-10}= \frac{2 \psi_{a}+2 \psi_{b}+2 \psi_{d 2}}{2}, \\
& \psi_{001}=\frac{m_{u} \psi_{c}}{2}
\end{aligned}
$$

and in terms of the specific free energy per unit cell at the interface with the substrate, where the adhesion energy is subtracted from the free surface energy, ${ }^{8}$

$$
\psi_{s}=\psi_{001}-m_{u} \psi_{\text {mol-sub }} .
$$

Here, as in Fig. $2, \psi_{a}, \psi_{b}, \psi_{d 1}$, and $\psi_{d 2}$ are the strengths of the nearest-neighbor interactions between two molecules in the direction indicated by the subscript. These strengths are defined as the absolute value of the intermolecular interaction energy at equilibrium geometry. Similarly, $\psi_{c}$ and $\psi_{\text {mol-sub }}$ are the interaction strengths between one molecule and a neighboring (001) layer of molecules and between one molecule and the substrate, respectively. Those formulas also apply for perylene provided $\psi_{a}, \psi_{b}, \psi_{d 1}$, and $\psi_{d 2}$ are interpreted as nearest-neighbor interactions between two dimers rather than between two individual molecules.

Note that it is assumed that the $a b$ plane of the crystal is parallel to the substrate surface. This is typically true for organic crystals of the herringbone or sandwich herringbone type (following the crystal structure classification in Ref. $9),{ }^{10-12}$ which have intense two-dimensional in-plane interactions. When those are grown on inert substrates-i.e., substrates that do not induce interactions with the adsorbing molecules that are stronger than their bulk crystal interactions - the $a b$ plane will indeed grow parallel to the substrate surface in order to maximize the intralayer interactions. A more formal way to verify the nucleus orientation on the substrate would be to calculate the minimum energy of nucleus formation of all possible orientations with respect to the substrate. 
a)

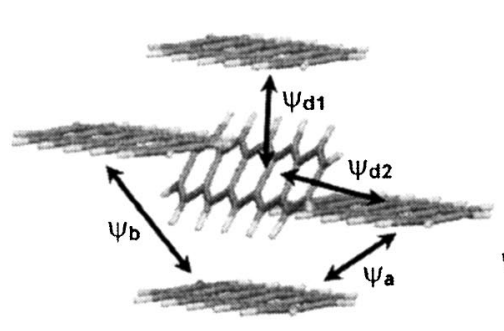

b)

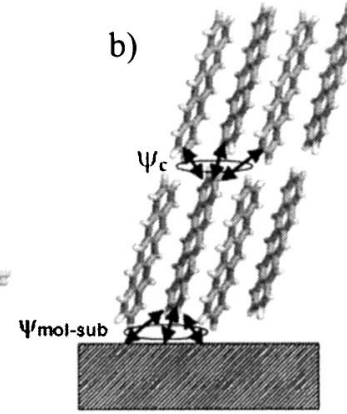

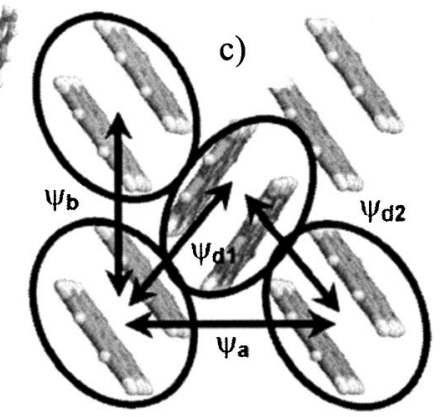

FIG. 2. (a) gives a view normal to the (001) plane and defines the intralayer nearest-neighbor interactions along the various directions between pentacene molecules, while (b) defines the interlayer interaction and the substrate-nucleus interaction. For perylene, all formulas apply when the intralayer interactions are defined as the interactions between dimers. The interlayer interactions for perylene are still defined per molecule.

Equation (1) shows that the formation of a nucleus is a competition between the energy released when transferring molecules from the supersaturated vapor inside the volume of an aggregate (first term) and the energy required to create phase-dividing surfaces (second term). A nucleus is formed by adding one molecule after another to an aggregate. For small aggregates, adding another molecule increases the surface effects, and the aggregate becomes more unstable. At a certain critical size with maximal instability, there are enough molecules that can arrange themselves to minimize edge effects such that the volume effect becomes dominant when adding new molecules to the aggregate; i.e., adding new molecules improves the stability of the aggregate and growth of the nucleus is favored more than its decay. In other words, the energy of nucleus formation can be regarded as an energy barrier which has to be overcome to create a stable nucleus.

The minimal size of this critical nucleus and therefore also its thermodynamically most stable shape can be found by differentiating Eq. (1) to $n_{a}, n_{b}, n_{c}, n_{d 1}$, and $n_{d 2}$ and setting those differentials to zero. ${ }^{8}$ The solution of that set of equations expresses the critical dimensions $n_{a}^{*}, n_{b}^{*}, n_{c}^{*}$, $n_{d 1}^{*}$, and $n_{d 2}^{*}$ in terms of the supersaturation $\Delta \mu$ and the nearest-neighbor interactions:

$$
n_{a}^{*}=\frac{n_{c}^{*}\left(2 \psi_{010}-\psi_{1-10}-\psi_{110}\right)}{\left(\psi_{001}+\psi_{s}-m_{u} n_{c} \Delta \mu\right)}
$$

$$
\begin{gathered}
n_{b}^{*}=\frac{n_{c}^{*}\left(2 \psi_{100}-\psi_{1-10}-\psi_{110}\right)}{\left(\psi_{001}+\psi_{s}-m_{u} n_{c} \Delta \mu\right)}, \\
n_{c}^{*}=\max \left(\frac{2\left(\psi_{001}+\psi_{s}\right)}{m_{u} \Delta \mu}, 1\right), \\
n_{d 1}^{*}=\frac{n_{c}^{*}\left(\psi_{1-10}-\psi_{010}-\psi_{100}\right)}{\left(\psi_{001}+\psi_{s}-m_{u} n_{c} \Delta \mu\right)}, \\
n_{d 2}^{*}=\frac{n_{c}^{*}\left(\psi_{110}-\psi_{010}-\psi_{100}\right)}{\left(\psi_{001}+\psi_{s}-m_{u} n_{c} \Delta \mu\right)} .
\end{gathered}
$$

Note that if some surface energies become too large, that surface will be excluded from the equilibrium shape of the nucleus (i.e., $n_{i}^{*}=0$, with $i=a, b, c, d 1$, or $d 2$ ), and Eq. (1) will have to be rewritten.

The height of the nucleus can physically not become less than one monolayer: i.e., $n_{c}^{*} \geqslant 1$. A nucleus with $n_{c}^{*}=1$ is called a two-dimensional (2D) nucleus. If $n_{c}^{*}>1$, the nucleus grows three-dimensionally (3D); i.e., the height of the critical nucleus in its thermodynamically most stable form scales with its lateral dimensions. The energy of formation of a critical nucleus then becomes

$$
\begin{gathered}
\Delta G_{3 D}^{*}=\frac{4\left(\psi_{001}+\psi_{s}\right)\left[2\left(\psi_{010}+\psi_{100}\right)\left(\psi_{110}+\psi_{1-10}\right)-2 \psi_{010}^{2}-2 \psi_{100}^{2}-\psi_{110}^{2}-\psi_{1-10}^{2}\right]}{\left(m_{u} \Delta \mu\right)^{2}}, \\
\Delta G_{2 D}^{*}=\frac{\left[2\left(\psi_{010}+\psi_{100}\right)\left(\psi_{110}+\psi_{1-10}\right)-2 \psi_{010}^{2}-2 \psi_{100}^{2}-\psi_{110}^{2}-\psi_{1-10}^{2}\right]}{m_{u} \Delta \mu-\left(\psi_{001}+\psi_{s}\right)}
\end{gathered}
$$

Three-dimensional nucleation is possible for all supersaturations $\Delta \mu>0$. Two-dimensional nuclation becomes possible only when a critical supersaturation $\Delta \mu_{\mathrm{cr}}$ has been reached:

$$
\Delta \mu_{\mathrm{cr}}=\frac{\psi_{001}+\psi_{s}}{m_{u}}=\psi_{c}-\psi_{\mathrm{mol}-\mathrm{sub}}
$$


TABLE I. Crystal structure data of the molecules and polymorphs considered in this study. The last two rows compare the heats of sublimation as predicted by the intermolecular interactions calculated with the MM3 force field with the experimentally determined heats of sublimation. The heat of sublimation $\Delta H_{\text {subl }}$ consists of the binding energy $\mathrm{BE} \approx \psi_{a}+\psi_{b}+\psi_{c}+\psi_{d 1}+\psi_{d 2}$, the translational and rotational energies (together $3 R T)$, and an additional $R T$ term to convert the internal energy $(\mathrm{BE}+3 R T)$ into enthalpy within the ideal gas approximation. For perylene, the binding energy is estimated as $\mathrm{BE} \approx\left(\psi_{a}+\psi_{b}+2 \psi_{c}+\psi_{d 1}+\psi_{d 2}\right.$ $\left.+\psi_{\text {dimer }}\right) / 2$. The temperature $T$ is chosen within the temperature range that was used to experimentally determine $\Delta H_{\text {subl }}$ for each molecule.

\begin{tabular}{lllll}
\hline \hline & \multicolumn{1}{c}{ Pentacene (1) } & Pentacene (2) & Tetracene & Perylene \\
\hline$a[\AA]$ & 7.90 & 6.265 & 6.057 & 11.277 \\
$b[\AA]$ & 6.06 & 7.786 & 7.838 & 10.826 \\
$c[\AA]$ & 16.01 & 14.511 & 13.010 & 10.263 \\
$\alpha[\mathrm{deg}]$ & 101.90 & 76.650 & 77.127 & 90.000 \\
$\beta[\mathrm{deg}]$ & 112.60 & 87.500 & 72.118 & 100.550 \\
$\gamma[\mathrm{deg}]$ & $85.80^{\mathrm{a}}$ & $84.610^{\mathrm{b}}$ & $85.792^{\mathrm{c}}$ & $90.000^{\mathrm{d}}$ \\
$\mathrm{BE}$ & 31.0 & 30.5 & 24.9 & 25.8 \\
$4 R T[\mathrm{kcal} / \mathrm{mol}]$ & $4.0(T=500 \mathrm{~K})$ & $4.0(T=500 \mathrm{~K})$ & 3.6 & 2.9 \\
& & & $(T=450 \mathrm{~K})$ & $(T=370 \mathrm{~K})$ \\
$\Delta H_{\text {subl }} \approx \mathrm{BE}+4 R T[\mathrm{kcal} / \mathrm{mol}]$ & 35.0 & 34.5 & 28.5 & 28.7 \\
$\Delta H_{\text {subl,experimental }}[\mathrm{kcal} / \mathrm{mol}]$ & $37.7^{\mathrm{e}}$ & $37.7^{\mathrm{e}}$ & $29.8^{\mathrm{e}}$ & $30.2^{\mathrm{f}}$ \\
& $37.0^{\mathrm{g}}$ & $37.0^{\mathrm{g}}$ & & \\
\hline \hline
\end{tabular}

${ }^{\mathrm{a}}$ Reference 21.

${ }^{\mathrm{b}}$ Reference 22 .

${ }^{\mathrm{c}}$ Reference 24 .

${ }^{\mathrm{d}}$ Reference 25 .

${ }^{\mathrm{e}}$ Reference 29 .

${ }^{\mathrm{f}}$ Reference 31.

${ }^{g}$ Reference 30 . This value is obtained by fitting the vapor pressures between 495 and $530 \mathrm{~K}$. The enthalpy of sublimation at $298.15 \mathrm{~K}$ was calculated to be $43.9 \mathrm{kcal} / \mathrm{mol}$, which seems rather high. This calculated value is obtained by extrapolating the experimentally determined enthalpy of sublimation over a $200 \mathrm{~K}$ temperature range, assuming a constant specific heat at constant pressure. In addition, the specific heat at constant pressure was not determined experimentally for pentacene, but extrapolated from experimental values for polycyclic hydrocarbons with a smaller number of benzene rings. Both extrapolations are questionable.

If $\Delta \mu_{\mathrm{cr}}$ is positive, the formation of a $3 \mathrm{D}$ nucleus requires less energy than 2D nucleation for supersaturations below a certain transition supersaturation $\Delta \mu_{\mathrm{tr}}$ (Ref. 8):

$$
\Delta \mu_{\mathrm{tr}}=2 \Delta \mu_{\mathrm{cr}}
$$

Below $\Delta \mu_{\text {tr }}, 3 \mathrm{D}$ nucleation will therefore be more probable than 2D nucleation. At $\Delta \mu_{\mathrm{tr}}$, the thermodynamically most favorable 3D nucleus is exactly one monolayer high: i.e., the thermodynamically most stable $3 \mathrm{D}$ nucleus is a $2 \mathrm{D}$ nucleus. At supersaturations higher than $\Delta \mu_{\text {tr }}$, the thermodynamically most stable 3D nucleus shape would be less than one monolayer high. Since this shape is physically not realizable, 2D nucleation will become most probable. However, depending on the substrate surface energy, $\Delta \mu_{\mathrm{cr}}$ can also be negative, in which case the energy of formation will always be lower for $2 \mathrm{D}$ than for 3D nucleation, and nucleation can already occur at undersaturation. ${ }^{8}$

Three-dimensional nuclei form ill-connected, isolated grains. When the height of the nucleus decreases and the lateral dimensions of the nucleus gain importance with respect to the height, the film grows more continuously. Ultimately, continuous films with two-dimensionally nucleated grains are formed. Therefore, if $\Delta \mu_{\mathrm{cr}}>0$, Eq. (7) can be used in a first approximation to distinguish between organic smallmolecular semiconductors that will grow continuous twodimensional films on inert substrates under favourable growth conditions-i.e., which are good candidates for charge-transport layers in OTFT's - and those that will grow noncontinuous films at similar growth conditions. Notice that the choice of substrate or surface treatment plays a very important role as well in determining the nucleation mode.

\section{MODEL PARAMETERS}

\section{A. Intermolecular interactions}

The strengths of the nearest-neighbor interactions $\psi_{a}$, $\psi_{b}, \psi_{c}, \psi_{d 1}, \psi_{d 2}$, and $\psi_{\text {dimer }}$ are estimated by molecular mechanic calculations with Allinger's MM3 force field, ${ }^{13}$ using the TINKER package. ${ }^{14}$ This force field has been specifically designed for quantitative studies of the structures and vibrational properties of aliphatic and aromatic hydrocarbons in the gas phase and crystals. One of the advantages of the MM3 force field over other molecular mechanic approaches (CHARMM, AMBER, etc.) is that it includes specific contribu- 
TABLE II. $\psi_{a}-\psi_{d 2}$ give the energy of the interactions between nearest neighbors in the direction of the subscript. For pentacene and tetracene, those nearest neighbors are individual molecules, while for perylene intralayer nearest neighbors (within the $a b$ plane) are dimers and interlayer nearest neighbors are individual molecules. To split a dimer into individual molecules, an energy $\psi_{\text {dimer }}$ needs to be supplied. Based on the nearest-neighbor interactions and unit cell parameters, it is possible to calculate the surface energies $\sigma$ of the surfaces that bind the nucleus. For comparison, surface energies computed in Ref. 34 have been included in between brackets. Also given is the number of molecules in a 2D nucleus at the transition supersaturation and its energy of formation, assuming no interaction between the nucleus and substrate.

\begin{tabular}{lcccc}
\hline \hline & Pentacene (1) & Pentacene (2) & Tetracene & Perylene \\
\hline$\psi_{a}[\mathrm{eV}]$ & 0.033 & 0.293 & 0.246 & 0.026 \\
$\psi_{b}[\mathrm{eV}]$ & 0.311 & 0.035 & 0.025 & 0.185 \\
$\psi_{c}[\mathrm{eV}]$ & 0.150 & 0.141 & 0.134 & 0.252 \\
$\psi_{d 1}[\mathrm{eV}]$ & 0.435 & 0.438 & 0.329 & 0.478 \\
$\psi_{d 2}[\mathrm{eV}]$ & 0.418 & 0.417 & 0.346 & 0.478 \\
$\psi_{\text {dimer }}[\mathrm{eV}]$ & - & - & - & 0.567 \\
$\sigma_{100}\left[\mathrm{meV} / \AA^{2}\right]$ & $4.8(4.8)$ & 6.6 & 5.9 & 4.6 \\
$\sigma_{010}\left[\mathrm{meV} / \AA^{2}\right]$ & $6.3(6.4)$ & 5.1 & 4.8 & 5.6 \\
$\sigma_{001}\left[\mathrm{meV} / \AA^{2}\right]$ & $3.2(3.1)$ & 2.9 & 2.8 & 4.2 \\
$\sigma_{110}\left[\mathrm{meV} / \AA^{2}\right]$ & $5.2(4.7)$ & 5.2 & 4.8 & 4.3 \\
$\sigma_{1-10}\left[\mathrm{meV} / \AA^{2}\right]$ & $5.0(4.8)$ & 5.5 & 5.0 & 4.3 \\
$\left\lceil m_{u} n_{a b}\left[\Delta \mu_{\text {tr }}\left(\psi_{\text {mol-sub }}=0\right)\right] 1\right.$ & 22 & 25 & 18 & 4 \\
$\Delta G_{2 D}^{*}\left[\Delta \mu_{\mathrm{tr}}\left(\psi_{\text {mol-sub }}^{*}=0\right)\right]$ & 3.29 & 3.43 & 2.30 & 0.88 \\
{$[\mathrm{eV}]$} & & & & \\
\hline \hline
\end{tabular}

tions for the out-of-plane deformation of aromatic rings. Also, in a MM3 treatment, the stretching and torsion force constants in conjugated segments are reparametrized iteratively at each step of a geometry optimization on $\pi$ densities computed using a self-consistent-field (SCF) procedure. ${ }^{15} \mathrm{By}$ virtue of its parametrization, this force field sustains very favorably the comparison with thorough quantum mechanical calculations involving exceedingly large basis sets and benchmark treatments of electron correlation. ${ }^{16}$ In the present work, in order to avoid $\pi$-SCF computations on systems with several thousands of atoms, we restrict such reparametrization to force constants computed within a single unit cell, considering that intermolecular interactions have negligeable impact on $\pi$ densities (an assertion which has already been checked in MM3 studies of catenanes, ${ }^{17}$ clusters of fullerenes, ${ }^{18}$ or model chains of poly-para-phenylenevinylene $^{19}$ ). Next, the experimentally determined unit cell parameters, listed in Table I, are used to build aggregates containing up to 2304 atoms, along with a replication of the force constants computed for a single unit cell. The structure of those aggregates is then relaxed into the minimum potential structure for the applied MM3 force field with the reparametrized force constants, while the experimental unit cell parameters are enforced by periodic boundary box conditions. Within this relaxed structure, the nonbonded interaction between pairs of nearest-neighbor molecules is calculated. The periodic boundary box conditions are required to select a specific polymorph structure.

Different polymorphs of pentacene have been interpreted in function of a gliding motion at the $\pi-\mathrm{H}$ (face to edge) overlap of neighboring inequivalent molecules. ${ }^{20}$ Without the periodic boundary box condition, the MM3 force field relaxes to an aggregate with crystal unit cell angles $\alpha \approx \beta$ $\approx 90^{\circ}$ and a $\pi$-H interaction which is close to maximal and with unit cell lengths that are predicted rather accurately. Two different polymorphs of pentacene have been calculated using the periodic boundary box conditions. The two polymorphs selected ${ }^{21,22}$ mimic most closely the polymorphs observed in pentacene thin films. ${ }^{10}$ These, moreover, have been shown to yield the most stable pentacene polymorphs. ${ }^{23}$ For tetracene, the crystal structure in Ref. 24 has been used. The $\alpha$ polymorph is selected for perylene, ${ }^{25}$ since the $\beta$ phase is stable only above $413 \mathrm{~K}$ or metastable. ${ }^{2}$ The unit cell parameters for all crystals under consideration are listed in Table I.

Table I also compares the estimated heats of sublimation based on calculations of internal energy differences with the MM3 force field with experimentally determined heats of sublimation. In straightforward analogy with the conversion of MM3 energies into heats (enthalpies) of formation within the ideal gas approximation, ${ }^{26-28}$ the heats (enthalpies) of sublimation are obtained in first approximation by adding thermodynamical corrections for free rotation $[(3 / 2) R T]$ and translations $[(3 / 2) R T]$ in the gas phase to the MM3 binding energies (BE's), along with the mechanical work $(R T)$ required for transferring one mole of molecules from the solid to the gas phase (with $R$ and $T$ the universal gas constant and the sublimation temperature, respectively). The binding energy of a molecule in the crystal is calculated with nearestneighbor interactions, neglecting interactions with more distant molecules. The Knudsen effusion method was used for the experimental determination of the heats of sublimation by measuring the temperature dependences of the vapor pressure. $^{29-31}$ Vapor pressure measurements of vapor-grown organic materials are not routine, however, and can vary substantially. ${ }^{32,33}$ For pentacene, the polymorph, of which the 
vapor pressure data was measured, was not identified. We have assumed the same vapor pressure data for both polymorphs, since our calculations show only minor differences in binding energies. The calculated heats of sublimation approach nicely the experimental results, and their energy order is predicted correctly.

Table II summarizes the nearest-neighbor interactions which are required to calculate the energy of nucleus formation and which were used to estimate the heat of sublimation in Table I. The nearest-neighbor interactions are responsible for over $95 \%$ of the interactions between an individual molecule and the rest of the aggregate, proving that secondnearest-neighbor interactions can effectively be neglected and that the "atomistic" approach describing the nucleus formation in terms of a limited number of discrete interactions is applicable.

Last, the specific free surface energies $\sigma$ per unit area can be calculated for each crystal surface based on the nearestneighbor interactions and the unit cell parameters and are listed in Table II:

$$
\sigma_{100}=\frac{\psi_{100}}{b c \sin \alpha}, \quad \sigma_{010}=\frac{\psi_{010}}{a c \sin \beta}, \quad \sigma_{001}=\frac{\psi_{001}}{a b \sin \gamma}, \quad \text { etc. }
$$

Despite the entirely different nature of the treatment which has been adopted here, the values obtained agree extremely well with total-energy calculations of the surface energies by means of density-functional theory, using first-principles pseudopotentials and a local density approximation: namely, the Ceperley-Alder exchange correlation energy function. ${ }^{34}$ The sole exception arises with $\sigma_{110}$, for which a discrepancy of $10 \%$ is seen.

From ratios of specific free surface energies, one can predict equilibrium crystal shapes according to Wulff's rule. ${ }^{8}$ In Fig. 1(a), we compare such a theoretical prediction for the equilibrium shape of a pentacene nucleus bounded by (010), (110), (100), (1-10), and (001) planes with an atomic force microscopic (AFM) picture of an isolated grain grown at very low supersaturation. The agreement between theory and experiment is very satisfactory. None of the surfaces in Table II has a high enough surface energy to be excluded from the equilibrium shape, validating the choice of the ten nucleus bounding surfaces in Eqs. (1) and (2). The calculated equilibrium crystal shape reproduces the main characteristics of isolated 3D pentacene grains grown at very low supersaturations, as the one in Fig. 1(b).

Since the heats of sublimation, which depend on the magnitude of the nearest-neighbor interactions, as well as the equilibrium shapes of nuclei, which depend on ratios of the nearest-neighbor interactions, are predicted well enough using the MM3 force field, we are confident that the values of the nearest-neighbor interactions are good approximations.

\section{B. Supersaturation}

Another important parameter which is required to explore the model described here is supersaturation, which depends on the vapor pressure of the material and on the growth conditions. For ambient vapor at a pressure $P_{v}$ and an infi- nitely large crystal at the same temperature $T$ and for small deviations from the equilibrium vapor pressure $P_{\infty}$, the supersaturation can be expressed as ${ }^{8}$

$$
\Delta \mu=\int_{P_{\infty}}^{P_{v}} \frac{\partial \mu_{v}}{\partial P} d P-\int_{P_{\infty}}^{P_{v}} \frac{\partial \mu_{c}}{\partial P} d P=R T \ln \left(\frac{P_{v}}{P_{\infty}}\right)
$$

by treating the vapor as an ideal gas, where $R$ is the universal gas constant. In a first approximation for typical OMBD conditions, the substrate can be thought of as a virtual infinitely large crystal at substrate temperature $T_{\text {sub }}$, and the ambient vapor pressure $P_{v}$ sensed by the substrate can be estimated from the deposition rate $\Phi$ as measured, e.g., by a quartz crystal monitor. ${ }^{35}$ Using experimentally determined vapor pressure data, ${ }^{29,31}$ the supersaturation can be written as a function of measurable quantities. Vapor pressure data can be expressed in the form

$$
\ln \left[P_{\infty}(T)\right] \approx A-\frac{\Delta H_{\text {subl }}}{R T},
$$

where $\Delta H_{\text {subl }}$ is the heat of sublimation and $A$ an experimentally determined term related to the entropy of sublimation. ${ }^{35,29}$ Note that $\Delta H_{\text {subl }}$ is approximately the energy required to separate a molecule from a half-crystal position and depends on the nearest-neighbor interactions as described above. The supersaturation is then given by

$$
\begin{aligned}
\Delta \mu & \approx R T_{\text {sub }} \ln \left(\frac{\sqrt{2 \pi M R T_{\text {sub }}} \Phi}{P_{\infty}\left(T_{\text {sub }}\right)}\right) \\
& \approx \Delta H_{\text {subl }}+R T_{\text {sub }}\left[\ln \left(\sqrt{2 \pi M R T_{\text {sub }}} \Phi\right)-A\right],
\end{aligned}
$$

where $M$ is the molecular mass and $\Phi$ is the deposition rate. Equation (11) is derived assuming that the molecules captured by the substrate thermalize and form a "gas" at the substrate temperature, which provides the molecules to form a nucleus. Strictly speaking, Eq. (11) is valid solely for 3D nucleation, because the supersaturation is referenced against the chemical potential of an infinitely large crystal and not to the chemical potential of the thin film. The chemical potential of the thin film is $\psi_{c}-\psi_{\text {mol-sub }}$ lower than the chemical potential of the infinitely large crystal. This difference can in first approximation be neglected for the materials under consideration, in comparison to the contributions of the other intermolecular interactions to the chemical potential, and Eq. (11) can then also be used to describe 2D nucleation.

\section{RESULTS AND COMPARISON WITH EXPERIMENTS}

\section{A. Pentacene}

We calculated the nucleation behavior of pentacene (2) in Fig. 3(a). Using only the experimentally determined vapor pressure data, we predict the OMBD conditions at which there is no supersaturation $(\Delta \mu=0)$. This condition is drawn as a solid line. At higher supersaturations, 3D nucleation is possible. Including also the calculated nearestneighbor interactions and assuming no substrate interactions $\left(\psi_{\text {mol-sub }}=0\right)$, we furthermore do predict the OMBD conditions that create the critical supersaturation $\Delta \mu_{\mathrm{cr}}$ (dashed 

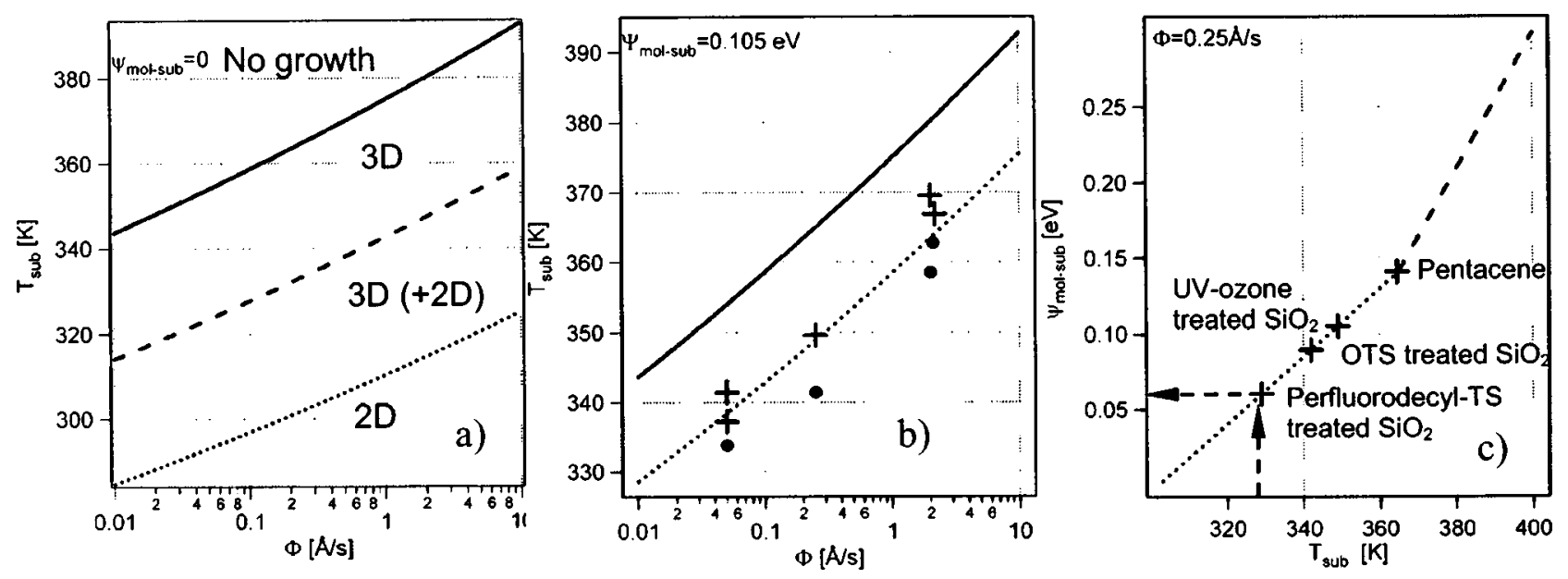

FIG. 3. (a) Growth modes for pentacene (2) assuming $\psi_{\text {mol-sub }}=0$. The solid line indicates zero supersaturation $(\Delta \mu=0)$ at the given flux and substrate temperature. Below the solid line, three-dimensional (3D) nucleation is possible. For growth conditions below the dashed line ( $\Delta \mu=\Delta \mu_{\mathrm{cr}}$ ), two-dimensional (2D) nucleation is possible. 2D nucleation becomes more likely than 3D nucleation only below the dotted line $\left(\Delta \mu=\Delta \mu_{\mathrm{tr}}=2 \Delta \mu_{\mathrm{cr}}\right)$. In a first approximation, only growth conditions below the dotted lines $\left(\Delta \mu>\Delta \mu_{\mathrm{tr}}\right)$ will give rise to continuous films. The size of the growth window for 3D nucleation is therefore fully determined by $\Delta \mu_{\text {tr }}$, depending strongly on $\psi_{\text {mol-sub }}$. Note that each line is also an equipotential contour for the energy of formation: at the solid lines, $\Delta G_{3 \mathrm{D}}^{*}$ becomes infinitely large, while at the dashed lines $\Delta G_{2 \mathrm{D}}^{*}$ becomes infinite [see Eq. (5)]. (b) Growth modes for pentacene (2) on a UV-ozone-treated $\mathrm{SiO}_{2}$ substrate. Solid circles show experimentally determined growth conditions at which the film appears continuous/2D, crosses are noncontinuous films/3D. The transition supersaturation is fitted with $\psi_{\text {mol-sub }}=0.105 \mathrm{eV}$. The window for 3D growth is significantly narrowed compared to the situation with $\psi_{\text {mol-sub }}=0 \mathrm{eV}$. Note that the substrate temperature in our experiments is calibrated to indicate the temperature at the surface of the sample. (c) Indicative values for $\psi_{\text {mol-sub }}$ for various surface treatments on $\mathrm{SiO}_{2}$, based on the experimentally determined transition substrate temperature from continuous to noncontinuous growth at a constant flux of $0.25 \AA / \mathrm{s}$. Using the theory presented here, the corresponding $\psi_{\text {mol-sub }}$ can then be calculated. If the substrate is a pentacene crystal, the film will always nucleate two dimensionally. For substrates with even higher molecule-substrate interactions, $\psi_{\text {mol-sub }}$ can be determined by finding $\Delta \mu_{\mathrm{cr}}$-i.e., the transition from growth (always $2 \mathrm{D}$ for those $\left.\psi_{\text {mol-sub }}\right)$ to no growth.

line). Above the critical supersaturation, $2 \mathrm{D}$ nucleation is possible. Under those same assumptions, the OMBD conditions leading to the transition supersaturation $\Delta \mu_{\mathrm{tr}}$ can be plotted. At $\Delta \mu_{\mathrm{cr}}<\Delta \mu<\Delta \mu_{\text {tr }}$ both 2D and 3D nucleation coexist in principle, yet 3D nucleation is far more probable. At $\Delta \mu>\Delta \mu_{\mathrm{tr}}, 2 \mathrm{D}$ nucleation is dominant. Since 3D nucleated films tend to grow ill-connected isolated grains, $\Delta \mu_{\text {tr }}$ can be in first approximation also be thought of as being the transition from noncontinuous to continuous films. In Fig. 3(a) it can be seen that the region for 3D growth at substrates with $\psi_{\text {mol-sub }} \approx 0$ is appreciable and within the range of easily accessible growth conditions. If $\psi_{\text {mol-sub }}$ increases, this 3D nucleation window will become smaller and vanishes at $\psi_{\text {mol-sub }}=\psi_{c}$. If $\psi_{\text {mol-sub }}>\psi_{c}, \Delta \mu_{\text {cr }}$ is negative as follows from Eq. (6) and the film will always nucleate two dimensionally, even at undersaturations $0>\Delta \mu>\Delta \mu_{\mathrm{cr}}$. Note that if $\psi_{\text {mol-sub }} \gg \psi_{c}$, the nucleus might change crystal orientation such that the (001) face is not parallel to the substrate anymore, and Eqs. (1)-(7) will need to be recalculated to find the new crystal orientation with minimal energy of nucleus formation.

It is clear from this analysis that $\Delta \mu_{\mathrm{tr}}=2\left(\psi_{c}-\psi_{\text {mol-sub }}\right)$ is the most important parameter determining the mode of nucleation and that for a given material the choice of substrate or surface modification allows some control over the mode of nucleation. In the present study, the $\Delta \mu_{\text {tr }}$ terms for given molecule-substrate pairs are estimated from the morphology of the pentacene films. Specifically, using atomic force microscopy (tapping mode) to identify the nucleation mode, it is possible to locate within a narrow window of growth conditions in substrate temperature versus flux plots (see, e.g., Fig. 3) the transition from a 2D nucleated film to a 3D nucleated film. Alternatively, this transition can often also be identified by means of an optical microscope, when examining the continuity of the film. As can be seen in Fig. 3 (b) for pentacene (2), this growth window accurately follows a constant supersaturation contour, in this case $\Delta \mu$ $\approx 0.072 \mathrm{eV}$ (or $\psi_{\text {mol-sub }} \approx 0.105 \mathrm{eV}$ when using the value from Table II for $\psi_{c}$ ) when growing pentacene in ultra high vacuum on a UV-ozone-treated $\mathrm{SiO}_{2}$ surface. The supersaturation fitting the $2 \mathrm{D}$ to $3 \mathrm{D}$ nucleated film transition is the transition supersaturation $\Delta \mu_{\mathrm{tr}}=2 \Delta \mu_{\mathrm{cr}}$ for the given molecule-substrate pair (for $\Delta \mu>0$; if $\Delta \mu<0$, the transition from film growth to no film growth will in principle be fitted by $\left.\Delta \mu_{\mathrm{cr}}\right)$. Note that some care is required in interpreting the morphology, since pentacene will nucleate two dimensionally on another pentacene layer $\left(\Delta \mu_{\mathrm{tr}}=0\right.$ if $\left.\psi_{\text {mol-sub }}=\psi_{c}\right)$. It is thus possible to have a $2 \mathrm{D}$ overgrowth on top of the $3 \mathrm{D}$ nucleation layer.

The procedure outlined above does not only provide the last unknown parameter for calculating the energy of nucleus formation, but also allows comparison of the growth of a molecular thin film on different surface treatments. A first indicative determination of the pentacene-substrate interaction for various surface treatments is given in Fig. 3(c). In light of this theory, the molecule-substrate interaction 


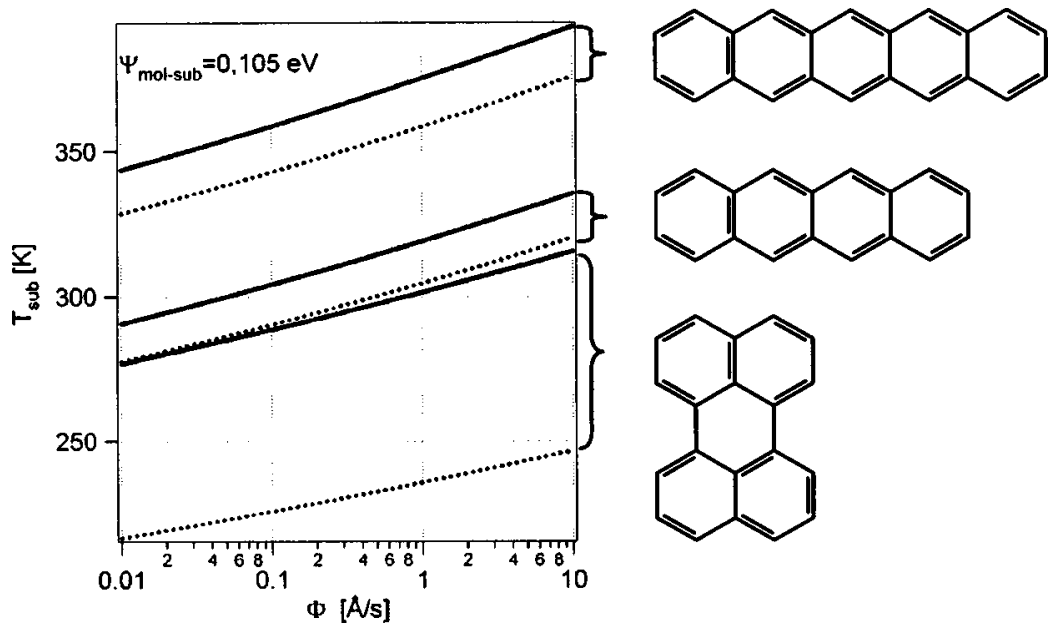

FIG. 4. The nucleation modes of pentacene, tetracene, and perylene for $\psi_{\text {mol-sub }}=0.105 \mathrm{eV}$. The solid lines demonstrate zero supersaturation for each material; the dotted lines are at transition supersaturation. The lines at zero supersaturation are solely determined by the vapor pressure for each material. Perylene has a much larger window for $3 \mathrm{D}$ growth, indicating that $\psi_{\text {mol-sub }}$ is significantly smaller than $\psi_{c}$. $\psi_{\text {mol-sub }}$ is a more relevant parameter than hydrophobicity or surface energy, ${ }^{36}$ yet will not be the only relevant surfacedependent parameter as soon as surface diffusion processes come into play. ${ }^{8}$

\section{B. Tetracene}

In addition to pentacene, we also calculated the nucleation behavior of two other organic small-molecular semiconducting materials: tetracene and perylene. The results are shown in Fig. 4, assuming $\psi_{\text {mol-sub }}=0.105 \mathrm{eV}$ for all three materials. Both pentacene and tetracene have similar interlayer nearestneighbor interactions $\psi_{c}$ and can be assumed to have similar

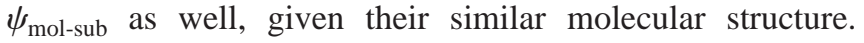
However, the heat of sublimation of tetracene is lower due to lower intralayer interactions. The intralayer interactions are governed by the $\pi-\pi$ and $\pi$-H interactions and approximately scale with the length of the molecule-i.e., the number of benzene rings. ${ }^{34}$ The standard vapor pressure of tetracene is higher than the vapor pressure for pentacene, ${ }^{29}$ and therefore the same supersaturation is reached at lower substrate temperatures and higher deposition rates. This picture qualitatively describes results published in literature. ${ }^{12}$

The window for 3D growth is relatively narrow for both pentacene and tetracene. The conditions to grow 3D tetracene films are more easily accessible than 3D pentacene films though, requiring less heating or cooling of the substrate at convenient deposition rates $(\sim 0.1-1 \AA / s)$. Yet we experimentally verified that $3 \mathrm{D}$ pentacene grains can be obtained at elevated substrate temperatures, as illustrated in Fig. 5(a).

Recall that the energy of formation of a nucleus is actually a barrier against nucleation and therefore is an important parameter in determining the nucleation rate. ${ }^{8}$ In all cases, the lowest $2 \mathrm{D}$ nucleation rates can be obtained at conditions close to the transition supersaturation. Although the transition supersaturation for pentacene and tetracene is very similar, the energy of formation of a tetracene nucleus at that supersaturation is smaller than the energy required to form a pentacene nucleus due to the lower intralayer interactions (Table II). In other words, nucleation rates for tetracene would be higher than for pentacene, favoring smaller grains for tetracene than for pentacene. Yet it should be kept in mind that the final grain size is not only determined by the energy of formation, but also by diffusion and desorption processes. $^{8}$

\section{Perylene}

Perylene has a heat of sublimation similar to tetracene. Yet the interlayer interactions $\psi_{c}$ are stronger for perylene than for tetracene. The rationale for this is that perylene is a bulkier molecule with a longer interaction width along the interlayer surface [i.e., one perylene molecule has four $\mathrm{C}-\mathrm{H}$ bindings pointing to a neighboring molecular (001) layer], while tetracene and pentacene only have a small width to interact [in this case, only two $\mathrm{C}-\mathrm{H}$ bindings are pointing to a neighboring molecular (001) layer]. Each atom or $\mathrm{C}-\mathrm{H}$ binding pointing out of the (001) surface is an interaction site for noncovalent interactions with atoms or bindings from neighboring surfaces. Thus a perylene crystal has a larger number of interaction sites at its (001) surface than pentacene or tetracene: perylene has $0.13 \mathrm{C}-\mathrm{H}$ bindings $/ \AA^{2}$ pointing out of the (001) surface, while pentacene has only $0.08 \mathrm{C}-\mathrm{H}$ bindings $/ \AA^{2}$ pointing out of its (001) surface. The strength of the interaction between two surfaces varies with the density of interaction sites at each of the surfaces. The interaction strength of molecules to a surface ( $\left.\psi_{\text {mol-sub }}\right)$ with given density of interaction sites is to a first instance proportional to the density of binding sites at the molecules. By the same reasoning, the interaction strength $\psi_{c}$ between molecular layers increases more than proportionally with the density of the binding sites at the molecules. Hence, $\psi_{c}$ will increase more than proportional with $\psi_{\text {mol-sub }}$ when increasing the density of the interaction sites of the (001) molecular surface while keeping the density of the interaction sites at the substrate constant. Therefore, the transition supersaturation is expected to increase superlinearly with the interaction width of the molecule, and thus it will be more difficult to grow a $2 \mathrm{D}$ perylene film on a given surface than to grow a $2 \mathrm{D}$ tetracene or pentacene film, which is consistent with the experimental observation in Fig. 5.

All our perylene films grow three dimensionally, which we attribute to a high $2 \mathrm{D}-3 \mathrm{D}$ transition supersaturation $\Delta \mu_{\text {tr }}$ 

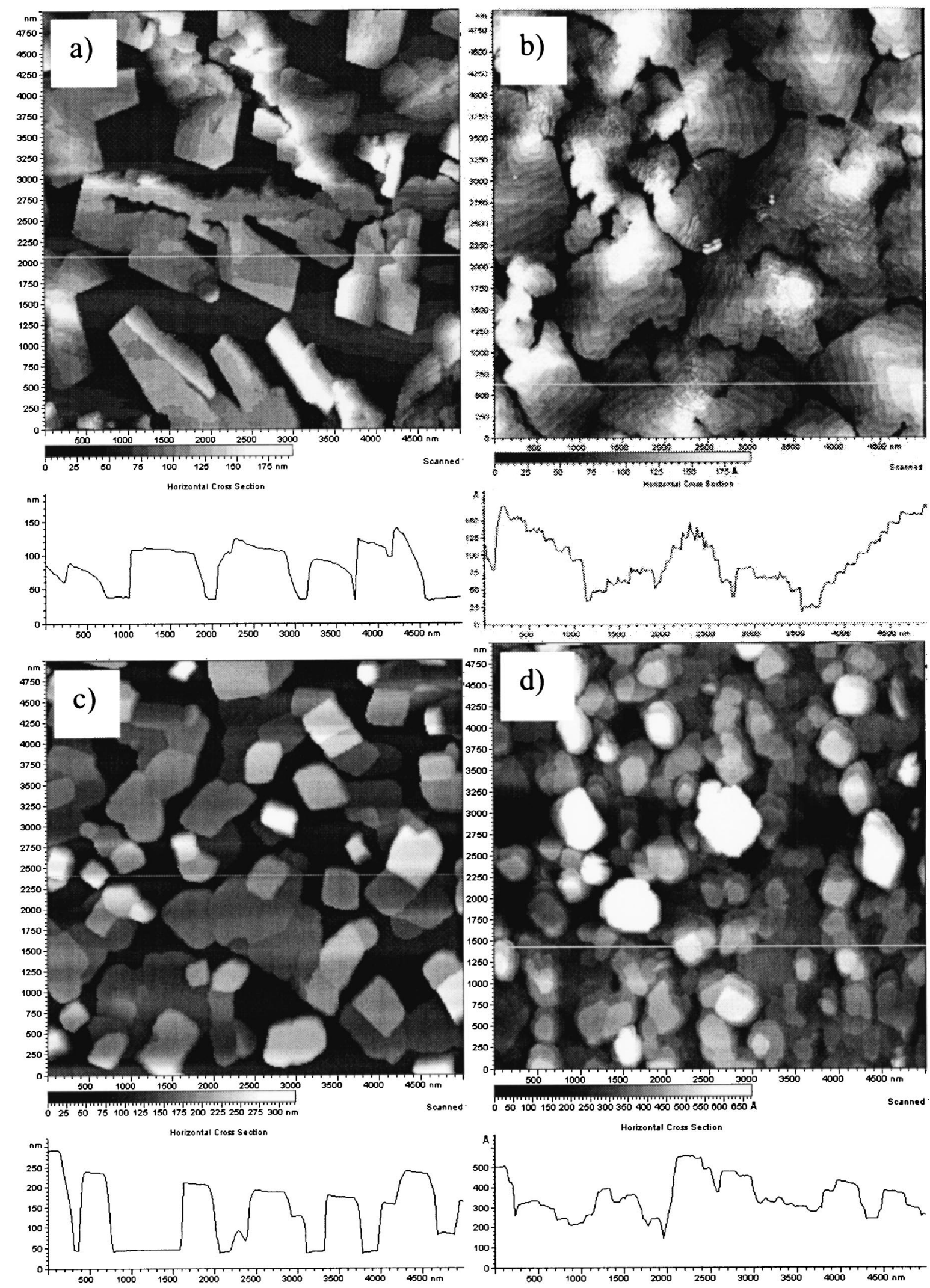

FIG. 5. Atomic force microscopy pictures, each $5 \times 5 \mu \mathrm{m}^{2}$ in size, of (a) $3 \mathrm{D}$ pentacene grains, grown at $T_{\text {sub }} \approx 337 \mathrm{~K}$ and $\phi$ $\approx 0.05 \AA / s$ on UV-ozone-treated $\mathrm{SiO}_{2}$. (b) $2 \mathrm{D}$ pentacene grains, grown at $T_{\text {sub }} \approx 334 \mathrm{~K}$ and $\phi \approx 0.05 \AA / \mathrm{s}$ on $\mathrm{SiO}_{2}$. The terraces have monolayer height. (c) 3D perylene grains, grown at $T_{\text {sub }} \approx 275 \mathrm{~K}$ (not calibrated) and $\phi \approx 0.3 \AA / s$ on iso-propyl-alcohol- (IPA-) treated $\mathrm{SiO}_{2}$. (d) 3D perylene grains, grown at $T_{\text {sub }} \approx 275 \mathrm{~K}$ (not calibrated) and $\phi \approx 1.3 \AA / \mathrm{s}$, on IPA-treated $\mathrm{SiO}_{2}$. The supersaturation in (d) is higher than in (c). The height scales of the cross sections are, respectively, $190 \mathrm{~nm}, 19 \mathrm{~nm}, 320 \mathrm{~nm}$, and $66 \mathrm{~nm}$. 
in addition to the high vapor pressure. The relatively high $\Delta \mu_{\text {tr }}$ means that $\psi_{\text {mol-sub }}$ is appreciably smaller than $\psi_{c}$ for the surfaces used [UV-ozone-treated $\mathrm{SiO}_{2}$, isopropanol(liquid-) treated $\mathrm{SiO}_{2}$, and octadecyltrichlorosilane- (OTS, vapor-) treated $\mathrm{SiO}_{2}$ ] and leads to a wide window for 3D nucleation, as expected from the above analysis. The high vapor pressure in combination with the wide window for $3 \mathrm{D}$ nucleation leads to the requirement of extensive substrate cooling and very high deposition rates to obtain $2 \mathrm{D}$ nucleation for the given surfaces. Figure 5(c) shows an example of 3D perylene grains. For comparison, Fig. 5(d) shows a perylene film with 3D grains, obtained at higher supersaturation. Since in the latter the supersaturation is higher and therefore the barrier against nucleus formation is lower, the nucleation rate is higher. Although the grains start to grow together and have crossed the percolation threshold, there are still voids. Field effect hole mobilities extracted from the OTFT saturation regime on those thin films never exceeded $0.003 \mathrm{~cm}^{2} / \mathrm{V} \mathrm{s}$ for purified perylene. The high transition supersaturation ultimately limits the maximum $2 \mathrm{D}$ grain size in a continuous film moreover to even smaller dimensions than for tetracene, since the energy of formation will be lower due to the high $\Delta \mu_{\text {tr }}$.

\section{CONCLUSIONS}

To conclude, we have interpreted the growth of pentacene, tetracene, and perylene on inert substrates using the microscopic theory of nucleation for the epitaxial growth of inorganic materials. These molecules crystallize in a layered structure with the layers parallel to the inert substrate and with a herringbone or sandwich herringbone arrangement within the layers, providing good $\pi$-orbital overlap for intralayer charge transport.

To form a continuous layer capable of transporting charge carriers, on a dielectric surface like in OTFT's, twodimensional nucleation is favored over 3D nucleation. The stronger the interlayer interactions and the smaller the molecule-substrate interactions, the larger the window to grow 3D grains, while the stronger the intralayer interactions, the more this window shifts to higher substrate temperatures-i.e., the more accessible the $2 \mathrm{D}$ growth conditions become.

As a rule of thumb, we suggest that molecules with a higher aspect ratio-i.e., a longer interaction length for in- tralayer interactions and a short interaction width for interlayer interactions-will have a larger region for $2 \mathrm{D}$ growth. Both $\psi_{c}$ and $\psi_{\text {mol-sub }}$ scale with the interaction width, and for shorter interaction widths the transition supersaturation will therefore decrease overall, resulting in a larger deposition window for $2 \mathrm{D}$ growth. Molecules with a higher aspect ratio will also have a higher energy of nucleus formation due to the higher intralayer interactions, as can be seen from Eqs. (5) and (7). The higher energy of nucleus formation will result in a higher barrier against nucleation and thus contributes to a lower nucleation rate, favoring larger grain sizes. Moreover, molecules with longer interaction length will have a larger heat of sublimation and will make the growth window for 2D growth more accessible without substrate cooling (stated differently, the 3D growth window will shift to higher substrate temperatures and lower fluxes). These kind of molecules-e.g., pentacene-will have the ability to form continuous films, potentially with large grains, at convenient growth conditions, and seem promising for charge transport applications as in OTFT's. Similarly, we predict that larger molecules with similar molecular and crystalline structures as perylene, like terrylene or quaterrylene, will have similar transition supersaturations as perylene, yet higher heats of sublimation and therefore lower vapor pressures, which will move the 2D growth window to more accessible deposition conditions. Perylene itself, on the other hand, appears to have a strong tendency for three-dimensional nucleation and tends to form ill-connected films with poor charge carrier properties. In any case, however, the molecule-substrate interaction is an important parameter controlling the growth mode.

\section{ACKNOWLEDGMENTS}

We gratefully acknowledge Professor Jean-Pierre Francois (Limburgs Universitair Centrum) for supporting this project, as well as Professor Norbert Karl (Universität Stuttgart) for providing purified perylene and interesting discussions. Also interaction with Wim Van Roy, Barundeb Dutta, Staf Borghs, and Stefan Nemeth (Imec) is highly appreciated. We thank the Cambridge Crystallographic Data Center for their service. S.V. and D.J. thank I.W.T.-Vlaanderen for financial support. M.D. acknowledges the F.W.O. and the Limburgs Universitair centrum (B.O.F.) for financial support.

${ }^{1}$ Thomas N. Jackson, Yen-Yi Lin, David J. Gundlach, and Hagen Klauk, IEEE J. Sel. Top. Quantum Electron. 4, 100 (1998); M. Matters, D. M. de Leeuw, M. J. C. M. Vissenberg, C. M. Hart, P. T. Herwig, T. Geuns, C. M. J. Mutsaers, and C. J. Drury, Opt. Mater. (Amsterdam, Neth.) 12, 189 (1999); P. F. Baude, D. A. Ender, M. A. Haase, T. W. Kelley, D. V. Muyres, and S. D. Theiss, Appl. Phys. Lett. 82, 3964 (2003).

${ }^{2}$ Norbert Karl, in Crystal and Solid State Physics, edited by Otfried Madelung, Landolt-Börnstein, New Series, Group III, Vol. $17 \mathrm{i}$ (Springer-Verlag Berlin, Heidelberg, New York, Tokyo,

1985), Chap. 12, p. 106.

${ }^{3}$ W. Warta, R. Stehle, and N. Karl, Appl. Phys. A: Solids Surf. 36, 163 (1985); Norbert Karl and Jörg Marktanner, Mol. Cryst. Liq. Cryst. Sci. Technol., Sect. A 355, 149 (2001).

${ }^{4}$ Y.-Y. Lin, D. J. Gundlach, S. F. Nelson, and T. N. Jackson, IEEE Electron Device Lett. 18, 606 (1997).

${ }^{5}$ D. J. Gundlach, Y.-Y. Lin, T. N. Jackson, S. F. Nelson, and D. G. Schlom, IEEE Electron Device Lett. 18, 87 (1997).

${ }^{6}$ Frank-J. Meyer zu Heringdorf, M. C. Reuter, and R. M. Tromp, Nature (London) 412, 517 (2001). 
${ }^{7}$ F. Biscarini, R. Zamboni, P. Samori, P. Ostoja, and C. Taliani, Phys. Rev. B 52, 14868 (1995); F. Biscarini, P. Samori, O. Greco, and R. Zamboni, Phys. Rev. Lett. 78, 2389 (1997).

${ }^{8}$ Ivan V. Markov, Crystal Growth for Beginners, 1st ed. (World Scientific, Singapore, 1995); I. Markov and S. Stoyanov, Contemp. Phys. 28, 267 (1987).

${ }^{9}$ Gautam R. Desiraju and A. Gavezzotti, Acta Crystallogr., Sect. B: Struct. Sci. 45, 473 (1989).

${ }^{10}$ I. P. M. Bouchoms, W. A. Schoonveld, J. Vrijmoeth, and T. M. Klapwijk, Synth. Met. 104, 175 (1999).

${ }^{11}$ Xiangdong Liu, S. H. Mohamed, J. M. Ngaruiya, Matthias Wuttig, and Thomas Michely, J. Appl. Phys. 93, 4852 (2003).

${ }^{12}$ D. J. Gundlach, J. A. Nichols, L. Zhou, and T. N. Jackson, Appl. Phys. Lett. 80, 2925 (2002).

${ }^{13}$ J.-H. Lii and N. L. Allinger, J. Am. Chem. Soc. 111, 8566 (1989); 111, 8576 (1989).

${ }^{14}$ J. W. Ponder, TINKER 3.1, Software Tools for Molecular Design, 1996.

${ }^{15}$ R. D. Brown and M. L. Heffernan, Aust. J. Chem. 12, 312 (1959).

${ }^{16}$ S. P. Kwasniewski, L. Claes, J.-P. Francois, and M. S. Deleuze, J. Chem. Phys. 118, 7823 (2003).

${ }^{17}$ M. S. Deleuze, F. Zerbetto, and D. A. Leigh, J. Am. Chem. Soc. 121, 2364 (1999); M. S. Deleuze, ibid. 122, 1130 (2000).

${ }^{18}$ M. S. Deleuze and F. Zerbetto, J. Am. Chem. Soc. 121, 5281 (1999).

${ }^{19}$ L. Claes, J.-P. Francois, and M. S. Deleuze, Chem. Phys. Lett. 339, 216 (2001).

${ }^{20}$ Christine Corinne Mattheus, Ph.D. thesis, Groningen University, 2002, Chap. 3, p. 45.

${ }^{21}$ R. B. Campbell, J. M. Robertson, and J. Trotter, Acta Crystallogr. 15, 289 (1962).
${ }^{22}$ T. Siegrist, C. Kloc, J. H. Schon, B. Batlogg, R. C. Haddon, S. Berg, and G. A. Thomas, Angew. Chem., Int. Ed. Engl. 40, 1732 (2001).

${ }^{23}$ Raffaele Guido Della Valle, Elisabetta Venutti, Aldo Brillante, and Alberto Girlando, J. Chem. Phys. 118, 807 (2003).

${ }^{24}$ D. Holmes, S. Kumaraswamy, A. J. Matzger, and K. P. C. Vollhardt, Chem.-Eur. J. 5, 3399 (1999).

${ }^{25}$ A. Camerman and J. Trotter, Proc. R. Soc. London, Ser. A 279, 129 (1964).

${ }^{26}$ U. Burkert and N. L. Allinger, Molecular Mechanics (American Chemical Society, Washington, D.C., 1982), Chap. 5, p. 174.

${ }^{27}$ N. L. Allinger, L. R. Schmitz, I. Motoc, C. Bender, and J. Labanowski, J. Am. Chem. Soc. 114, 2880 (1992).

${ }^{28}$ D. W. Hertz and N. L. Allinger, Tetrahedron 35, 3 (1979).

${ }^{29}$ Nobuko Wakayama and Hiroo Inokuchi, Bull. Chem. Soc. Jpn. 40, 2267 (1967).

${ }^{30}$ C. G. De Kruif, J. Chem. Thermodyn. 12, 243 (1980).

${ }^{31}$ Hiroo Inokuchi and Hideo Akamatu, Solid State Phys. 12, 93 (1961).

32 James S. Chickos and William E. Acree, J. Phys. Chem. Ref. Data 31, 537 (2002).

${ }^{33}$ Ch. Kloc and R. A. Laudise, J. Cryst. Growth 193, 563 (1998).

${ }^{34}$ John E. Northrup, Murilo L. Tiago, and Steven G. Louie, Phys. Rev. B 66, 121404(R) (2002).

${ }^{35}$ Milton Ohring, The Materials Science of Thin Films, 1st ed. (Academic Press, London, 1992), Chap. 3, p. 81.

${ }^{36}$ Laura L. Kosbar, Christos D. Dimitrakopoulos, and Debra J. Mascaro, in Symposium C: Electronic, Optical and Optoelectronic Polymers and Oligomers, edited by Ghassan E. Jabbour and Niyazi Serdar Sariciftci, Mater. Res. Soc. Symp. Proc. No. 665 (Materials Research Society, Pittsburgh, 2001), p. C10.6. 